\title{
Distributed series resistance effects in solar cells
}

\author{
Nielsen, Lars Drud
}

Published in:

I E E E Transactions on Electron Devices

Link to article, DOI:

10.1109/T-ED.1982.20784

Publication date:

1982

Document Version

Publisher's PDF, also known as Version of record

Link back to DTU Orbit

Citation (APA):

Nielsen, L. D. (1982). Distributed series resistance effects in solar cells. I E E E Transactions on Electron Devices, 29(5), 821-827. https://doi.org/10.1109/T-ED.1982.20784

\section{General rights}

Copyright and moral rights for the publications made accessible in the public portal are retained by the authors and/or other copyright owners and it is a condition of accessing publications that users recognise and abide by the legal requirements associated with these rights.

- Users may download and print one copy of any publication from the public portal for the purpose of private study or research.

- You may not further distribute the material or use it for any profit-making activity or commercial gain

- You may freely distribute the URL identifying the publication in the public portal

If you believe that this document breaches copyright please contact us providing details, and we will remove access to the work immediately and investigate your claim 


$$
X_{c}=\lim _{s \rightarrow W_{i}} \frac{e^{y_{s}}-1}{y_{s}} \int_{0}^{s} \frac{x / X_{0}}{e^{x / X_{0}}} d x .
$$

The solved integral yields

$$
X_{c}=\lim _{s \rightarrow W_{i}} X_{0} \frac{e^{y_{s}}-1}{y_{s}}\left[1-\frac{1+s / X_{0}}{e^{s / X_{0}}}\right] .
$$

This result is simplified to

$$
X_{c}=X_{0} \frac{e^{y}-1}{y} \text {. }
$$

\section{ACKNOWLEDGMENT}

The author is grateful to VALVO-RHW der Philips GmbH for supporting the publication and to Dr. W. Steinmaier for his encouragement.

\section{REFERENCES}

[1] B. H. Yun and P. C. Arnett, "Silicon nitride trap properties as revealed by charge-centroid measurements on MNOS devices,"
Appl. Phys. Lett., vol. 26, pp. 94-96, Feb. 1975.

[2] P. C. Arnett, "Transient conduction in insulators at high fields," J. Appl. Phys., vol. 46, pp. 5236-5243, Dec. 1975.

[3] K. Lehovec, "Charge distribution in the nitride of MNOS memory devices," J. Electron. Mater, vol. 6, pp. 77-93, Mar. 1977.

[4] K. Lehovec and A. Fedotowsky, "Transient charge and current distribution in the nitride of MNOS devices," IEEE Trans. Electron Devices, vol. ED-24, pp. 536-540, May 1977.

[5] - "Charge-centroid in MNOS devices," $J$. Appl. Phys., vol. 48, pp. 2955-2960, July 1977.

[6] M. Schumacher et al., "A lower band gap charge storage structure,"Rev. Phys. Appl., vol. 13, pp. 829-832, Dec. 1979.

[7] A. EL-Dessouky and P. Balk, "Degradation of MIOS nonvolatile memory structures," Rev. Phys. Appl., vol. 13, pp. 833-836, Dec. 1979.

[8] —, "Charge distribution in MAOS nonvolatile memory structures," Thin Solid Films, vol. 75, pp. 195-197, Feb. 1981.

[9] B. H. Yun, "Measurement of charge propagation in $\mathrm{Si}_{3} \mathrm{~N}_{4}$ films," Appl. Phys. Lett., vol. 25, pp. 330-342, Sept. 1974.

[10] K. Lehovec et al., "MNOS charge versus centroid determination by staircase charging," IEEE Trans. Electron Devices, vol. ED-25, pp. 1030-1036, Aug. 1978.

[11] C. Svensson and K. Lundstrom, "Trap-assisted charge injection in MNOS structures," J. Appl. Phys., vol. 44, pp. 4657-4663, Oct. 1973.

[12] A. EL-Dessouky, "Schaltverhalten von MIOS Festwertspeichern," Ph.D. dissertation, Technical University of Aachen, Aachen, Germany, 1979, unpublished.

\title{
Distributed Series Resistance Effects in Solar Cells
}

\author{
LARS DRUD NIELSEN
}

\begin{abstract}
A mathematical treatment is presented of the effects of one-dimensional distributed series resistance in solar cells. A general perturbation theory is developed, including consistently the induced spatial variation of diode current density and leading to a first-order equivalent lumped resistance of one third the total sheet resistance. For the case of diode characteristics of exponential type and distributed resistance of arbitrary size, unified numerical results are presented for both illuminated and dark characteristics. At high forward dark currents, the distributed series resistance is shown to cause an effective doubling of the "diode quality factor."
\end{abstract}

\section{INTRODUCTION}

$\mathrm{S}$ OLAR CELLS are inherently low-impedance electrical power generators. Large-area silicon cells may, under normal insolation, produce amperes of current at maximum

Manuscript received August 24, 1981. This work was supported by the Commission of the European Communities under Contract ESC-R020-DK(G).

The author is with Physics Laboratory III, Technical University of Denmark, DK-2800 Lyngby, Denmark. voltages of around half a volt. Therefore, a minimization of series resistance effects is of vital importance for the achievement of high solar cell efficiencies.

Early attention to the series resistance problems was given by, for instance, Wolf [1], whose treatment of solar cell limitations included design criteria for contact grids under the assumption of uniform current generation. Wysocki [2] investigated, experimentally and by specific numerical examples, the series resistance problem for GaAs cells, influenced by lumped contact resistance as well as distributed sheet resistance. Later, Handy [3] attempted to include the complicated two-dimensional sheet resistance problem in a complete model of solar cell series resistance, but apparently failed to interpret properly his results in terms of an equivalent series resistance. More recently, Boone and Van Doren [4] have studied a distributed model with exponential type diode characteristics, however, again under the erroneous assumption of uniform current generation.

The aim of this work is to present a consistent treatment of 
(a)

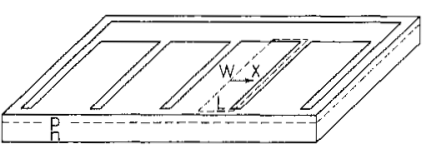

(b)

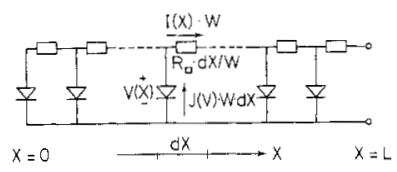

Fig. 1. (a) Example of a one-dimensional distributed series resistance problem. Sheet current in the $\mathrm{p}$-type top layer of the solar cell is assumed to flow perpendicularly to the grid lines $(W>L)$. (b) Onedimensional model of the "half unit field" (dotted rectangle) in (a).

the problem of distributed series resistance in solar cells. The model described in the following section is based on the concrete example of sheet resistivity in the top layer of a solar cell, which may be of particular importance for cells operating in concentrated sunlight, or cells with low top layer conductivity, such as: ion-implanted cells, metal-insulator-semiconductor cells, inversion-layer cells, or cells based on various alternative semiconductor materials. However, the results are applicable to other sources of distributed resistance as well.

The following section sets up a one-dimensional model of a solar cell with distributed series resistance and the fundamental differential equations governing the spatial variation of diode voltage and sheet current density. In Section III, a general perturbation theory is developed, from which the first-order results can be interpreted as arising from an equivalent lumped resistance. Section IV presents a numerical treatment of the important cases of exponential diode characteristics, with distributed series resistance of arbitrary magnitude. Illuminated and dark situations are treated separately. The latter case is formulated in a completely unified way, whereas the solutions to the illuminated situations can be described by a single parameter. A special asymptotic solution for the dark characteristic at high forward currents is dealt with in Section V, and final conclusions are drawn in Section VI.

The actual influence of series resistance on the solar cell "fill factor" and efficiency will depend on the absolute values of open-circuit voltage and will not be touched upon here. Illustrative examples may be found, for instance, in Hovel's textbook [5, Secs. 3.B.2 and 4.A.4].

\section{One-Dimensional Model of Distributed SERIES RESISTANCE}

The present study of distributed series resistance problems will be based on a purely one-dimensional model as shown in Fig. 1(b). The model might, for instance, represent half a "unit field" between the grid lines of a p-n-junction solar cell as shown in Fig. 1(a).

The sheet resistivity (ohms per square) of the p-layer is denoted $R_{\square}$. Current flow $I$ (amperes per meter) in the sheet is assumed to be parallel to the $X$-axis. The diode current density $J$ (amperes per square meter) depends on the local diode voltage $V$ and includes the photogenerated current. $W$ denotes the width of the unit field (length of a grid line) perpendicular to the $X$-axis. $X=0$ corresponds to a symmetry line with zero sheet current, whereas the output terminal (grid line) is posi- tioned at $X=L$. Signs for current and voltage are chosen to be positive for the device operating as an active solar cell.

With different interpretations of the involved symbols, the model of Fig. 1(b) might as well describe the problems of distributed metallic resistance along a grid line or contact bar, or generally: any kind of loss-affected parallel connection of elemental modules to form a linear chain with terminals at one end.

As $d X$ in Fig. 1(b) approaches zero, the following pair of coupled first-order differential equations is obtained for determination of $V(X)$ and $I(X)$ :

$$
\begin{aligned}
& \frac{d V}{d X}=-R_{\square} I \\
& \frac{d I}{d X}=J(V) .
\end{aligned}
$$

Boundary conditions may be formulated as

$$
\begin{gathered}
V(0)=V_{0} \\
I(0)=0 .
\end{gathered}
$$

With $V_{0}$ (diode voltage at the left end of the array) as a param. eter, solutions may be obtained for the voltage and current distributions, $V(X)$ and $I(X)$, and-especially-for the terminal quantities $V_{L}=V(L)$ and $I_{L}=I(L)$.

Only in cases of extremely trivial intrinsic characteristics, $J(V)$, do (1), (2) have analytical solutions. In practice, one is, therefore, left with the possibilities of approximative or numerically based solutions.

\section{Perturbational Approach}

The sheet resistivity $R_{\square}$ is used as a perturbation parameter. At any position $X(0 \leqslant X \leqslant L)$ the voltage and sheet current density are expanded in power series of $R_{\square}$

$$
\begin{aligned}
& V(X)=\sum_{n=0}^{\infty} R_{\square}^{n} \cdot V_{(n)}(X) \\
& I(X)=\sum_{n=0}^{\infty} R_{\square}^{n} \cdot I_{(n)}(X) .
\end{aligned}
$$

Due to the boundary conditions (3), (4), all expansion coefficients $V_{(n)}(X)$ and $I_{(n)}(X)$ will vanish at $X=0$, except $V_{(0)}(0)$ $=V_{0}$. Obviously, the unperturbed case $\left(R_{\square}=0\right)$ will correspond to a constant voltage $V_{0}$ over the entire cell. So, the intrinsic characteristic $J(V)$ is expanded as a Taylor series around $V=V_{0}$

$$
J(V)=J\left(V_{0}+\delta V\right)=\sum_{m=0}^{\infty} \frac{J^{(m)}}{m !} \cdot \delta V^{m}
$$

where

$$
J^{(m)}=\left.\frac{d^{m} J(V)}{d V^{m}}\right|_{V=V_{0}} \quad\left(J^{(0)}=J\left(V_{0}\right)\right) .
$$

From (5), the voltage perturbation is 
TABLE I

Results of Perturbation Calculations up to Second Order

\begin{tabular}{|l|l|l|}
\hline Order & Voltage & Sheet current \\
\hline 0 & $R_{\square}^{n} \cdot V_{(n)}(x)$ & $R_{\square}^{n} \cdot I_{(n)}(x) \cdot W$ \\
\hline 1 & $-\frac{1}{2} R_{\square} J(0) x^{2}$ & $-\frac{1}{6} R_{\square} J^{(0)} J^{(1)} x^{3} \cdot W$ \\
2 & $-\frac{1}{24} R_{\square}^{2} J^{(0)} J^{(1)} x^{4}$ & $\frac{1}{120} R_{\square}^{2}\left[3 J(0)^{2} J^{(2)}-J(0)_{J}(1)^{2}\right] x^{5} \cdot W$ \\
\hline
\end{tabular}

$$
\begin{aligned}
& J^{(0)}=J\left(\mathrm{v}_{0}\right), \quad J^{(1)}=\left.\frac{\mathrm{dJ}}{\mathrm{dV}}\right|_{\mathrm{V}=\mathrm{v}_{0}}, \quad \mathrm{~J}^{(2)}=\left.\frac{\mathrm{d}^{2} \mathrm{~J}}{\mathrm{d \textrm {v } ^ { 2 }}}\right|_{\mathrm{V}=\mathrm{v}_{0}} \\
& \delta V(X)=V(X)-V_{0}=\sum_{n=1}^{\infty} R_{\square}^{n} \cdot V_{(n)}(X) .
\end{aligned}
$$

Substitution of (9) into (7) and rearrangement of terms lead to

$$
J(X)=\sum_{n=0}^{\infty} R_{\square}^{n} \cdot J_{(n)}(X)
$$

where $J_{(n)}(X)$ are constructed from the voltage perturbation functions $V_{(n)}(X)$ and the derivatives $J^{(m)}$ of the intrinsic characteristic, as listed in the Appendix.

Substitution of (5), (6), and (10) into (1) and (2) leads to the following recursive formulas for determination of $V_{(n)}(X)$ and $I_{(n)}(X)$ :

$$
\begin{gathered}
\frac{d}{d X} V_{(n)}(X)=-I_{(n-1)}(X) \\
\frac{d}{d X} I_{(n)}(X)=J_{(n)}(X) .
\end{gathered}
$$

Solutions to these equations are shown in Table I, up to secondorder perturbation. Calculations to higher orders are left to the reader. The functional dependences on position $X$ appear from the table. Values at the cell terminal (grid line) are obtained for $X=L$.

It is of particular interest to notice that, for small values of sheet resistivity, the distributed series resistance model may be approximated by a simple equivalent circuit with a lumped series resistance. The proof, and the determination of the equivalent lumped resistance $R_{S}$, follow from the first-order perturbations

$$
\begin{aligned}
\delta V_{L} & =-\frac{1}{2} J^{(0)} R_{\square} L^{2} \\
\delta I_{L} \cdot W & =-\frac{1}{6} J^{(0)} J^{(1)} R_{\square} L^{3} W .
\end{aligned}
$$

The voltage increment (13) is here to be interpreted as arising partly from the current increment (14) via the dynamical diode resistance $\left[f^{(1)} \cdot L \cdot W\right]^{-1}$ and partly from the voltage drop of the total (zero-order) diode current $J^{(0)} \cdot L \cdot W$ across the lumped resistance $R_{S}$. With the sign conventions in mind, this yields

$$
\delta V_{L}=\frac{\delta I_{L} \cdot W}{J^{(1)} \cdot L \cdot W}-R_{S} \cdot J^{(0)} \cdot L \cdot W
$$

which, by insertion of (13), (14), can be seen to be satisfied for

$$
R_{S}=\frac{1}{3} R_{\square} \cdot L / W
$$

independently of the value $J^{(0)}$ and derivative $J^{(1)}$ of the intrinsic diode characteristic.

By calculation to first order in $R_{\square}$, it may be verified that the above expression for $R_{S}$ also leads to the correct resistive power dissipation in the sheet. Actually, this criterion has been used, without justification of the detailed consequences regarding the current-voltage characteristic, to define an equivalent lumped resistance, as seen, for instance, in a recent review paper by Hall [6].

The accuracy of the lumped resistance approximation can only be judged in actual cases. A good accuracy should at least be expected as long as the second-order corrections in Table I are small compared to the first-order corrections. This criterion will depend on the diode characteristic, including illumination level, the magnitude of $R_{S}$, and the load conditions. An example is shown in Fig. 3 and is commented in the following section.

From (16), it is seen that the effective series resistance equals one third the total sheet resistance along the device. In the otherwise detailed (two-dimensional) analysis by Handy [3] this factor of $\frac{1}{3}$ has obviously been overlooked. The apparent agreement with experiments may be ascribed to the circumstance that sheet resistance was only one out of several contributions to the total series resistance of the investigated cells. The more recent work by Boone and Van Doren [4] comes out with a factor of $\frac{1}{2}$ instead of $\frac{1}{3}$. The discrepancy arises from an inconsistent neglect of the first-order current correction (14). Also Wolf [1], in his early treatment of grid optimization, assumes uniform current generation and gets to factors of $\frac{1}{2}$ on the metallic grid line resistance as well as the diffused layer resistance.

Wysocki [2] reported on numerical calculations of effiency degradation of intrinsically 19-percent efficient GaAs cells, due to both contact resistance and sheet resistance. His results seem to confirm a reasonable validity of (16) down to resulting efficiencies below 10 percent.

\section{Numerical Approach}

\section{A. Illuminated Case}

In order to carry through numerical calculations of distributed series resistance effects, it will be necessary to make some assumptions concerning the diode characteristic $J(V)$, hopefully without severe restrictions on general interest and applicability. Anyway, a broad variety of solar cell characteristics conform, within the relevant range of voltages, to an expression of the type

$$
J(V)=J_{S C}-J_{D} \cdot\left[\exp \left(V / V_{T}\right)-1\right] .
$$

$J_{S C}$ is the photoinduced short-circuit current density, $J_{D}$ the 
constant of diode dark-current density, and $V_{T}$ a characteristic voltage (equal to $k T / e$ for an ideal p-n-junction diode). The -1 in the brackets may, for the present purpose, safely be neglected, leading to

$$
J(V)=J_{S C}-J_{D} \cdot \exp \left(V / V_{T}\right)
$$

with an open-circuit voltage of

$$
V_{O C}=V_{T} \cdot \ln \left(J_{S C} / J_{D}\right) \text {. }
$$

Besides the sheet resistivity and the geometry of the cell, three new parameters from (18) are now being involved in the model. A properly unified treatment of this multiparameter problem can be obtained by introduction of the following transformations:

$$
\begin{aligned}
\Delta v & =\left(V-V_{O C}\right) / V_{T} \\
i & =\alpha I / J_{S C} \\
x & =\alpha X
\end{aligned}
$$

where

$$
\alpha=\sqrt{R_{\square} J_{S C} / V_{T}}
$$

is a characteristic reciprocal length. The dimensionless quantities $\Delta v, i$, and $x$ represent, respectively: voltage deviation from open-circuit voltage, sheet current density, and position on a scale from zero to

$$
l=\alpha L=\sqrt{R_{\square} J_{S C} / V_{T}} \cdot L .
$$

Substitution of (18)-(23) into the fundamental equations (1), (2) leads to

$$
\begin{aligned}
\frac{d \Delta v}{d x} & =-i \\
\frac{d i}{d x} & =1-\exp (\Delta v) .
\end{aligned}
$$

The boundary conditions (3), (4) transform to

$$
\begin{aligned}
\Delta v(0) & =\Delta v_{0}=\left(V_{0}-V_{O C}\right) / V_{T} \\
i(0) & =0 .
\end{aligned}
$$

The numerical exercise is now reduced to an integration of the unified differential equations (25), (26) with the boundary value $\Delta v_{0}(27)$ as the only parameter. The direct solutions are $\Delta v(x)$ and $i(x)$, from which the terminal quantities can be obtained as

$$
\begin{aligned}
& V_{L}=V_{O C}+V_{T} \cdot \Delta v(l) \\
& I_{L}=J_{S C} \cdot i(l) / \alpha .
\end{aligned}
$$

The output current per unit area of the cell is

$$
J_{\mathrm{av}}=I_{L} / L=J_{S C} \cdot j_{\mathrm{av}}(l)
$$

where

$$
j_{\mathrm{av}}(x)=i(x) / x
$$

By means of a small Fortran program, a tabulation was produced of the functions $\Delta v(x), i(x)$, and $j_{\mathrm{av}}(x)$, with $\Delta v_{0}=$ $-4.0,-3.8, \cdots, 1.0$. These boundary conditions cover the
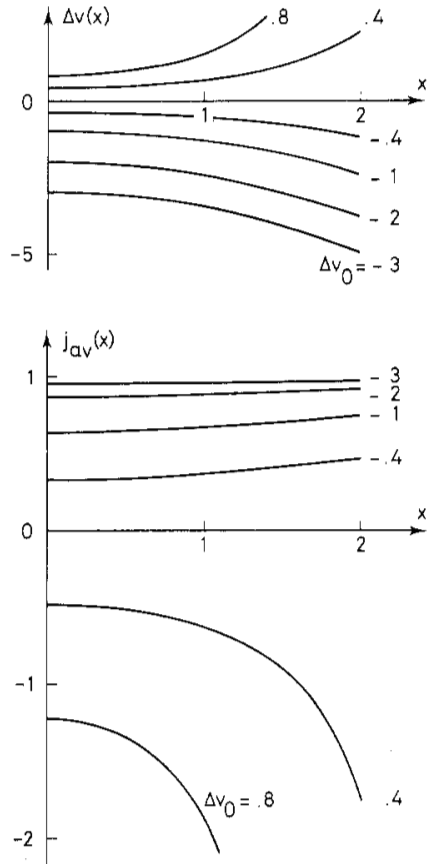

Fig. 2. Unified curves for illuminated diode voltage and average current density versus distance. $x$ : distance from left end of the cell, multiplied by $\alpha=\sqrt{R_{\square} J_{S C} / V_{T}} \cdot \Delta v$ : diode voltage, measured from open-circuit point in units of $V_{T}(20) . \Delta v_{0}$ : boundary value at $x=0(27)$. $j_{\text {av }}$ : average diode current density from 0 to $x$ in units of $J_{S C}((31),(32))$.

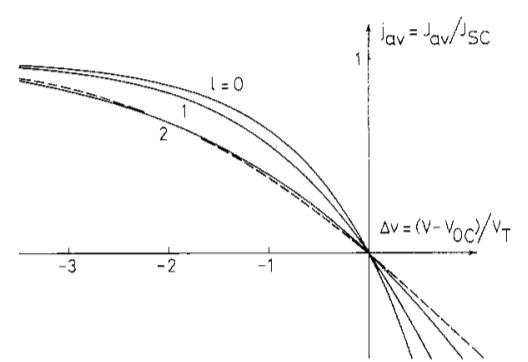

Fig. 3. Unified illuminated current-voltage characteristics with varying sheet resistance degradation. The parameter $l$ is given by (24). The dotted line shows a lumped resistance approximation corresponding to $l=2\left(r_{S}=\frac{4}{3}(33)\right)$.

range of interest for practical operation of solar cells and for experimental determination of series resistance according to the procedure described by Handy [3]. Integrations were carried out from $x=0$, corresponding to the lossless case, and up to $x \equiv 2$, corresponding to cases with pronounced inhomogeneity of diode voltage. Selected results are shown in Fig. 2 for $\Delta v(x)$ and $j_{\mathrm{av}}(x)$. Fig. 3 displays the normalized characteristics, $j_{\mathrm{av}}$ versus $\Delta v$, for cells with different sheet resistivity degradation (24): $l=0,1,2$.

The corresponding lumped resistance values, according to (16) and normalized by $V_{T} /\left(J_{S C} L W\right)$, are

$$
r_{S}=\frac{1}{3} l^{2} \text {. }
$$

For comparison, the lumped resistance approximation for $l=2$ $\left(r_{S}=\frac{4}{3}\right)$ is shown in Fig. 3 by a dotted line. The deviations from the exact calculations are surprisingly small for this rather extreme example, actually less than 0.1 on the horizontal scale 
within the voltage range from the open-circuit point to $\Delta v=$ -4. An explanation can be found in the circumstance that the second-order corrections in Table I, for characteristics of the type assumed in (18), tend to cancel each other for voltages below $\Delta v=\ln 1.5\left(V \equiv V_{O C}+0.4 \cdot V_{T}\right)$.

\section{B. Dark Case}

The substitutions used above can obviously not be applied to the case of a dark diode with $J_{S C}=0$. Equation (18) degenerates to

$$
J(V)=-J_{D} \exp \left(V / V_{T}\right)=-J_{0} \exp \left(\left(V-V_{0}\right) / V_{T}\right)
$$

where $V_{0}$ and $J_{0}$ are chosen as the actual diode voltage and current density at $X=0$. Transformation to dimensionless variables is now accomplished through

$$
\begin{aligned}
\Delta v & =\left(V-V_{0}\right) / V_{T} \\
i & =\beta I / J_{0} \\
x & =\beta X
\end{aligned}
$$

where

$$
\beta=\sqrt{R_{\square} J_{0} / V_{T}} .
$$

The parameter $\beta$ and the normalized length of the cell

$$
l=\beta L=\sqrt{R_{\square} J_{0} / V_{T}} \cdot L
$$

are now bias dependent through $J_{0}$.

Substitution of (34)-(38) into (1), (2) leads to

$$
\begin{aligned}
\frac{d \Delta v}{d x} & =-i \\
\frac{d i}{d x} & =-\exp (\Delta v) .
\end{aligned}
$$

The boundary conditions (13), (14) transform to

$$
\begin{aligned}
\Delta v(0) & =0 \\
i(0) & =0 .
\end{aligned}
$$

As the bias parameters $\left(V_{0}, J_{0}\right)$ now enter solely via the scaling factor $\beta$ (38), only one integration has to be performed to yield a unified pair of functions, $\Delta v(x)$ and $i(x)$, describing the voltage and sheet current variations along the cell. The integration was again carried out from $x=0$ to $x \equiv 2$, where numerical problems now become severe, due to a very rapid rise of $\Delta v(x)$. Results are shown in Fig. 4 for $\Delta v(x)$ and $i(x)$. Hereafter, the terminal quantities are obtained as

$$
\begin{aligned}
& V_{L}=V_{0}+V_{T} \cdot \Delta v(l) \\
& I_{L}=J_{0} \cdot i(l) / \beta .
\end{aligned}
$$

A unified current-voltage characteristic may be constructed with $l$ as an intermediate parameter, keeping in mind the interrelations between $l, J_{0}$, and $V_{0}$. From (39), the current density at the left end of the cell may be expressed as

$$
J_{0}=J_{00} \cdot l^{2}
$$

where

$$
J_{00}=V_{T} /\left(R_{\square} \cdot L^{2}\right)
$$

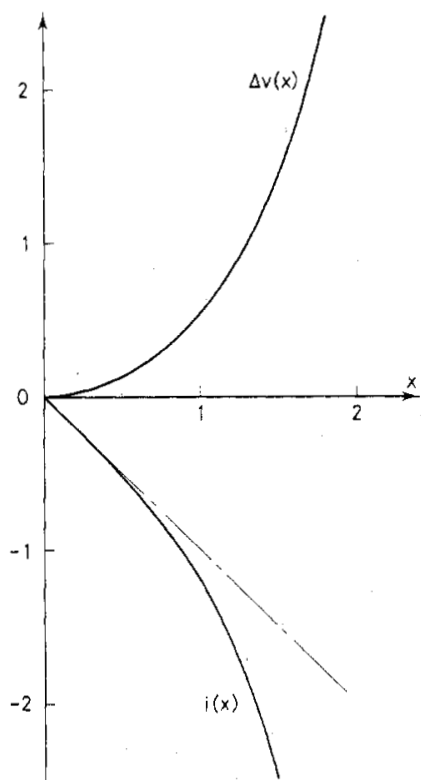

Fig. 4. Unified curves for dark diode voltage and sheet current density versus distance. $x$ : distance from left end of the cell, multiplied by $\beta=$ $\sqrt{R_{\square} J_{0} / V_{T}} . \Delta v$ : diode voltage, measured from $V_{0}$ in units of $V_{T}(35)$. $i$ : sheet current density in units of $J_{0} / \beta(36)$. $\left(V_{0}, J_{0}\right)$ : diode voltage and current density at the left end of the cell (34).

is a characteristic current density. The output current per unit area of the cell follows from (45) and (46) as

$$
J_{\mathrm{av}}=I_{L} / L=J_{0} \cdot i(l) / l=J_{00} \cdot j_{\mathrm{av}}(l)
$$

where

$$
j_{\mathrm{av}}(l)=i(l) \cdot l \text {. }
$$

The diode voltage at the left end of the cell may be expressed by means of (34) and (46) as

$$
V_{0}=V_{T} \cdot \ln \left(J_{0} / J_{D}\right)=V_{T} \cdot\left[\ln \left(J_{00} / J_{D}\right)+2 \ln l\right]
$$

where the first term in the brackets does not depend on $l$. Equation (44) then yields

$$
V_{L}=V_{T} \cdot\left[\ln \left(J_{00} / J_{D}\right)+v(l)\right]
$$

with

$$
v(l)=2 \ln l+\Delta v(l)
$$

Obviously, $v=0$ would correspond to a (uniform) current density of $J_{00}$, if the sheet resistance were removed for a while.

Fig. 5 shows a logarithmic plot of the unified characteristic $j_{\mathrm{av}}$ versus $v$. For comparison, the lumped resistance approximation, according to (16), is drawn by a dotted line. It is readily seen that this curve may be obtained by replacing (52) with

$$
v=\ln \left|j_{\mathrm{av}}\right|+\frac{1}{3}\left|j_{\mathrm{av}}\right| .
$$

From Fig. 5 it is seen that the calculated characteristic $(a)$ at low currents $\left(\left|j_{\mathrm{av}}\right| \ll 1\right)$ follows the ideal characteristic $(b)$ : $j_{\mathrm{av}}=-\exp (v)$. The deviations from ideality around $\left|j_{\mathrm{av}}\right|=1$ are properly accounted for by the lumped resistance model (c). At higher current levels, the behavior is strongly different from that of the lumped model, in contrast to the examples of 


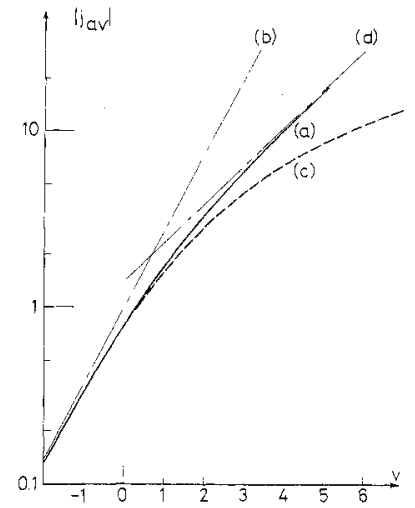

Fig. 5. Unified dark current-voltage characteristics: (a) numerical calculations, $(b)$ ideal characteristic, $(c)$ lumped resistance approximation, (d) asymptotic expression (56). $v$ represents, apart from an additive constant, the terminal voltage in units of $V_{T}(51)$. $j_{\text {av }}$ represents the terminal current per unit area of the cell, normalized to $V_{T} /\left(R_{\square} L^{2}\right)$ ((47), (48)).

illuminated characteristics in the normal operating regime (Fig. 3). Finally, it will be shown in the following section that an asymptotic solution of the type: $\exp (v / 2)$, as indicated by (d) in Fig. 5, will exist for $\left|j_{\mathrm{av}}\right| \gg 1$.

\section{Asymptotic BeHaVior OF THE DARK CHARACTERISTIC}

From the normalized fundamental equations (40), (41), the following general relation is obtained by elimination of $d x$ :

$$
i \cdot d i=\exp (\Delta v) \cdot d(\Delta v) .
$$

Integration with the boundary conditions (42), (43) yields

$$
i^{2}(x)=2 \cdot[\exp (\Delta v(x))-1]
$$

which is valid for all values of $x$. For $x \gg 1 \Delta v(x)$ increases extremely rapidly, and the -1 in the brackets may comfortably be disregarded. Making use of this approximation and applying (49) and (52), one obtains the following relationship for large values of the parameter $l$ :

$$
\begin{aligned}
\left|j_{\mathrm{av}}\right| & =|i(l) \cdot l| \equiv \sqrt{2} \cdot \exp (\Delta v(l) / 2) \cdot l \\
& =\sqrt{2} \cdot \exp (\Delta v(l) / 2) \cdot \exp [(v(l)-\Delta v(l)) / 2] \\
& =\sqrt{2} \cdot \exp (v / 2)
\end{aligned}
$$

This asymptotic behavior is illustrated in Fig. 5 by the line (d). It should be noticed that the bendover from one exponential relation to another corresponds to an effective doubling of the characteristic voltage parameter: $V_{T} \rightarrow 2 V_{T}$, no matter what was the initial value of $V_{T}$. So, it seems possible that the presence of a dominant distributed series resistance might, in some cases, be the real explanation of the high values of "diode quality factor" ( $n=e V_{T} / k T \geqslant 2$ ) observed in many experiments, as for instance in those reviewed by Hovel [5, sec. 3.C].

In order to get a feeling of the importance of the phenomena described above, a numerical example should be considered. Let a solar cell be characterized by the parameters: $V_{T}=26$ $\mathrm{mV}, R_{\square}=100 \Omega$, and $L=0.2 \mathrm{~cm}$ (half grid line spacing). According to (47), the critical forward current density $J_{00}$ then amounts to only $6.5 \mathrm{~mA} / \mathrm{cm}^{2}$. The equivalent lumped series resistance $R_{S}$ (16) will be $1.33 \Omega$ for a $1-\mathrm{cm}^{2}$ cell. If the cell is now illuminated to $J_{S C}=26 \mathrm{~mA} / \mathrm{cm}^{2}$, the dimensionless length parameter $l$ of (24) will be equal to 2. From Fig. 3 it is seen that this case is still very well accounted for by the lumped resistance approximation.

From this discussion, it should be expected that measurements of the effective series resistance will depend strongly on the experimental procedure applied. Examples of such discrepancies were early reported by Wolf and Rauschenbach [7] .

Finally, it should be mentioned that other types of large-area semiconductor components might be markedly influenced by the distributed series resistance effects described here. Sheet resistivities can, of course, be much lower when optical considerations are not in question, but practical current densities will often be higher by orders of magnitude.

\section{CONCLUSIONS}

The effects of one-dimensional distributed series resistance in solar cells have been studied by perturbational and numerical methods. It has been demonstrated that these phenomena cannot be consistently handled without taking into account the spatial variation of diode current density. A proper firstorder approximation to the distributed resistance problem leads to an equivalent lumped resistance equal to one third of the total sheet resistance along the cell.

This first-order approximation is shown to be fully adequate for a description of the series resistance degradation of illuminated characteristics under most normal operating conditions. As far as concerns the forward dark-current characteristic, however, pronounced deviations from the lumped model set in at moderate current levels. At high currents, the impact of the distributed resistance turns out to be an effective doubling of the diode quality factor, $n=e V_{T} / k T$. These effects might have implications for other large-area devices as well.

The conclusions concerning the adequacy of the lumped resistance approximation suggest experimental determination of effective series resistance to be based on the recording of different illuminated characteristics, as described by Handy [3], rather than on measurements of the dark characteristic at high forward currents. Conversely, the latter procedure may furnish valuable diagnostic information about the nature of the dominant contribution to the total series resistance of a solar cell.

\section{APPENDIX}

A List of the First Six Expansion Functions for the Perturbed Diode Current Density (10)

$$
\begin{aligned}
J_{(0)}= & J^{(0)}=J\left(V_{0}\right) \\
J_{(1)}= & J^{(1)} V_{(1)} \\
J_{(2)}= & J^{(1)} V_{(2)}+\frac{1}{2} J^{(2)} V_{(1)}^{2} \\
J_{(3)}= & J^{(1)} V_{(3)}+J^{(2)} V_{(1)} V_{(2)}+\frac{1}{6} J^{(3)} V_{(1)}^{3} \\
J_{(4)}= & J^{(1)} V_{(4)}+J^{(2)}\left[V_{(1)} V_{(3)}+\frac{1}{2} V_{(2)}^{2}\right] \\
& +\frac{1}{2} J^{(3)} V_{(1)}^{2} V_{(2)}+\frac{1}{24} J^{(4)} V_{(1)}^{4} \\
J_{(5)}= & J^{(1)} V_{(5)}+J^{(2)}\left[V_{(1)} V_{(4)}+V_{(2)} V_{(3)}\right] \\
& +\frac{1}{2} J^{(3)}\left[V_{(1)}^{2} V_{(3)}+V_{(1)} V_{(2)}^{2}\right] \\
& +\frac{1}{6} J^{(4)} V_{(1)}^{3} V_{(2)}+\frac{1}{120} J^{(5)} V_{(1)}^{5} .
\end{aligned}
$$


$V_{(n)}$ and $J_{(n)}$ are functions of position, $0 \leqslant X \leqslant L$. The coefficients $J^{(m)}$ are derivatives of the intrinsic diode characteristic at $V=V_{0}(8)$.

\section{REFERENCES}

[1] M. Wolf, "Limitations and possibilities for improvement of photovoltaic solar energy converters," Proc. IRE, vol. 48, p. 1246, 1960.

[2] J. J. Wysocki, "The effect of series resistance on photovoltaic solar energy conversion," RCA Rev., vol. 22, p. 57, 1961.

[3] R. J. Handy, "Theoretical analysis of the series resistance of a solar cell," Solid-State Electron., vol. 10, p. 765, 1967.

[4] J. L. Boone and T. P. Van Doren, "Solar-cell design based on a distributed diode analysis," IEEE Trans. Electron Devices, vol. ED-25, p. $767,1978$.

[5] H. J. Hovel, "Solar cells," in Semiconductors and Semimetals, vol. 11, R. K. Willardson and A. C. Beer, Eds. New York: Academic Press, 1975.

[6] R. N. Hall, "Silicon photovoltaic cells," Solid-State Electron., vol. 24 , p. $595,1981$.

[7] M. Wolf and H. Rauschenbach, "Series resistance effects on solar cell measurements," Advanced Energy Conversion, vol. 3, p. 455 , 1963.

\title{
An Area-Variable MOS Varicap and Its Application in Programmable TAP Weighting of CCD Transversal Filters
}

\author{
A. B. BHATTACHARYYA AND HANS WALLINGA
}

\begin{abstract}
A new three-terminal MOS varicap is proposed where the terminal capacitors are made voltage variable not by the modulation of depletion width but by changing the area of inversion under the gate. An MOS capacitor realized on silicon with an impurity gradient along the surface provides the control on the area of inversion because the gate threshold voltage is determined by the doping concentration at the surface.

The inhomogeneous doping along the surface is implemented making use of the lateral diffusion from a doped oxide surface. Fabrication details of the capacitor compatible with n-channel silicon gate technology are presented. The $C--V$ relationship for the terminal capacitors is simulated by a piecewise model and agreement with measured results is shown.

The Area-Variable MOS Varicap (AVMOSV) is used in implementing an electrically programmable CCD filter with variable TAP weighting. Computer simulation shows considerable promise of area-variable capacitors in TAP weight control and transversal filter realization. Preliminary performance characteristics of a programmable CCD filter are presented.
\end{abstract}

Manuscript received May 8,1981; revised October 7, 1981. Financial support for this paper was made possible by the Department of Electronics, Indian Institute of Technology, New Delhi, India, the Government of India, and The Twente University of Technology, Enschede, The Netherlands.

A. B. Bhattacharyya is with the Centre for Applied Research in Electronics, Indian Institute of Technology, Hauz Khas, New Delhi, 110016 India.

H. Wallinga was with the Solid State Electronics Group, Department of Electrical Engineering, The Twente University of Technology, 7500 AE Enschede, PB 217, The Netherlands. He is now on a visiting assignment at General Electric Company, Schenectady, NY 12301.

\section{INTRODUCTION}

V OLTAGE VARIABLE CAPACITORS or varicaps have found a wide range of applications such as voltage controlled oscillators, tunable integrated circuits, FM deviators [1], and specific tuning elements [2], [3], etc. Generally, such capacitors are two-terminal elements realized with a $\mathrm{p}-\mathrm{n}$ junction or MOS structure and their capacitance variation with voltage depends on the principle that the depletion width either at the $p-n$ junction or at the semiconductor-insulator interface in the deep depletion mode can be controlled electrically. Since the capacitance is directly proportional to the area and inversely proportional to the depletion width, conventional capacitances with a given area decrease with increasing voltage. There are, however, specific needs where more functional flexibility is required such as in a high-frequency switching application when a three-terminal structure is suitable [4].

On-chip MOS varicaps have been used recently for introducing a compact circuit for programming the TAP weights of a CCD filter electronically [5], [6]. In such implementations, the sense gates of a CCD are loaded by varicaps and a parallel sense capacitor. The part of the CCD image-signal charge through the sense capacitor depends on the ratio of the variable MOS capacitor value and fixed sense capacitor. This part or fraction is controlled or programmed by voltage. The advantage of capacitive weighting is the low power dissipation and smaller chip area compared to the structures where a combination of a floating sense gate and a buffer circuit performs 